\title{
Size Effect in Waveguide Coupled Whispering Gallery Mode Disk Resonators
}

\author{
Michael Rosenblit, ${ }^{1}$ Peter Horak, ${ }^{2}$ and Ron Folman ${ }^{1}$ \\ ${ }^{1}$ Department of Physics and Ilse Katz Center for Meso- and Nanoscale Science and Technology, \\ Ben Gurion University of the Negev, P.O.Box 653, Be'er Sheva 84105, Israel \\ ${ }^{2}$ Optoelectronics Research Centre, University of Southampton, Southampton SO17 1BJ, UK
}

\begin{abstract}
We investigate the bound and evanescent fields of the optical whispering gallery modes which are supported by a toroid microcavity and which may be used for a wide range of applications. Results of simulations using finite-difference time domain solutions of Maxwell's equations are compared with semi-analytical solutions based on coupled mode theory. Key parameters such as resonance frequencies, transmittance characteristics, coupling efficiencies, and bending/scattering losses are analyzed as a function of experimental variables such as size, distance, and fabrication roughness. Finally, the feasibility of single-atom detection is discussed.
\end{abstract}

Keywords: microdisk resonator, atom detection, WGM

\section{INTRODUCTION}

Micro resonators are a promising system for a wide range of applications because of the existence of highquality optical modes, where light is trapped within very small volumes. Their spectral properties can be exploited in various fields ranging from telecommunication $[1,2]$ to biological/chemical sensors [3] as well as in fundamental research. Of specific interest is the potential contribution of such devices to the fast emerging field of quantum technology (QT), which may again serve as an enabling technology for both fundamental science and applicative research [4]. In particular, micro resonators can be used to enhance the interaction of light with single atoms or ions, which have been identified as a promising system for storage of quantum information.

Recently reported progress in the manufacturing of high Q dielectric microdisk resonant structures [5] motivates the development of compact and integrable devices. Of specific interest is the wafer-based manufacturing of resonators where a good control of the physical characteristics can be achieved during fabrication enabling for example extremely small mode volumes as well as accurate alignment with other elements such as micro traps. Furthermore, a wafer based device may allow for more complex functions such as tunability to be integrated.

In this paper we investigate the external fields of optical whispering gallery modes which are supported by a toroid microcavity and which may be used in QT.

This work is organized as follows. In Sec. II we describe the system under consideration. Section III deals with the different numerical and analytical methods which we apply to examine the disk resonator structures. In Sec. IV we discussed loss mechanisms and presented results of our simulation. Subsequently, we discussed experimental considerations such as tunability (Sec. V). We investigate the feasibility of single-atom detection using the calculated optical modes in Sec. VI. Finally, we conclude in Sec. VII.

\section{SYSTEM DESIGN}

The basic system under discussion consists of a circular plate (disk) and a linear waveguide, as shown in Fig. 1. This minimal system can be used as an integrated part of a larger chip containing several optical, electronical, and micromechanical devices. As an example, we will discuss single-atom detection as a possible application of this device for QT purposes in Sec. VI.

The waveguide couples light into and out of the disk. In a realistic setup, both waveguide ends will be attached to optical fibers. In order to optimize power transfer between the waveguide and a single-mode fiber, the mode overlap at the interface has to be maximized. This requires waveguide dimensions of $9 \times 9$ to $12 \times 12$ microns in the refractive index range of $1.454-2.17$ for wavelengths around $780 \mathrm{~nm}$. In this case, best coupling efficiencies of the order of $96-98 \%$ can be achieved. For best mode matching with the microdisk modes, the waveguide width has to be reduced to about 0.3 to $1.2 \mu \mathrm{m}$ using adiabatic tapers, as shown in Fig. 1. 


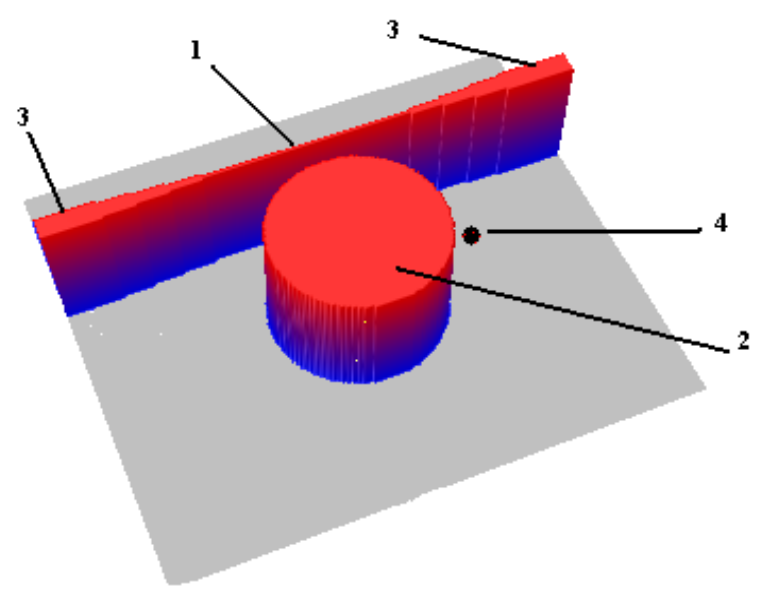

FIG. 1: The structure under consideration. The evanescent wave from the slab waveguide (1) is coupled into the disk (2) and back through a small gap between them. The adiabatic waveguide tapers (3) serve to couple light back and forth from an optical fiber (not shown) to the waveguide (of any needed size). An interaction with an external particle (4) may be initiated by positioning the particle on the disk side.

The disk itself supports a large range of resonant modes. Here, we are mainly interested in the low-loss modes traveling along the disk edge in the form of whispering gallery modes (WGM). While most of the mode energy is confined within the disk, a small part of the mode exists outside the disk as an evanescent field, and it is here that an atom can interact with the light. This external particle-light coupling changes the optical properties of the disk mode, which subsequently changes the intensity and/or phase of the light at the output of the linear waveguide. These changes can then be measured to infer the presence of the external particle.

In the horizontal direction the linear waveguides and the disk are bordered by the air $\left(n_{\text {clad }}=1.0\right)$. In our calculations we assumed structures made of fused silica with $n_{c}=1.454$ at a wavelength of $780 \mathrm{~nm}$. In the vertical direction the structure may be more complicated with several layers in order to give good mode confinement.

We investigate the resonance behavior of the microdisk cavity. The general scheme is simple: light enters the system through the waveguide and couples into the microdisk at point 1 in Fig. 1 . The intensity in the disk is significantly increased depending on the coupling efficiency, and on intrinsic and other loss mechanisms. The disk supports both clockwise and counterclockwise propagating modes. Direct coupling excites the clockwise mode but backscattering due to side-wall roughness can excite the counterpropagating mode.

The refractive index (RI) difference between core and cladding defines the bending losses for a given disk diameter, which together with the external loss mechanisms defines the quality factor. The balance between RI, disk diameter, waveguide width, gap width between the straight waveguide and disk resonator, and the surface quality of the disk determine the WGM characteristics.

\section{SIMULATION FRAMEWORK AND METHODS}

A number of numerical and analytical tools have been utilized to investigate the optical properties of such systems, for example coupled mode theory (CMT) [8, 9], scattering matrix theory [10], finite element [11], and finite difference time domain (FDTD) methods [12]. In this work we will use FDTD simulations which provide rigorous numerical results but which are rather time-consuming and therefore not adapt to scan large parameter ranges. We will thus resort to a semi-analytical CMT as a fast tool for a detailed design parameter analysis. These two methods complement one another and give a powerful tool for the investigation of micro resonators. In particular, we are interested in the system characteristics dependent on disk diameter, waveguide width, gap 
width between the linear waveguide and the disk resonator, and the surface quality of the disk.

\section{A. Finite difference time domain calculations}

The first method we use to investigate the resonance behavior of the microdisk is by FDTD simulations. Here, Maxwell's equations are discretized in space and time, and the time evolution of the electromagnetic fields is numerically calculated on this grid. To obtain high accuracy it is necessary to make the cells much smaller than the optical wavelength, which leads to long calculation times, in particular for a three-dimensional (3D) model.

In two-dimensional simulations it is possible to do direct FDTD calculation for relatively large disks of diameter $>50 \mu \mathrm{m}$. In 3D, simulations are only feasible for small disk diameters. However, comparisons of $2 \mathrm{D}$ and 3D simulations for small disk diameters have shown reasonable agreement. In the rest of this paper, we will thus restrict ourselves to simplified 2D calculations, where the disk and waveguide modes are calculated for a geometry infinitely extended in the vertical $(y)$ direction. The 3D modes are assumed to be simple slices of thickness $d_{y}$ of these infinite modes. For the calculations presented in this work we always assume $d_{y}=5 \mu \mathrm{m}$. We will also limit the calculations to electric fields polarized along the $y$ direction, i.e., to TE modes only.

In this case, Maxwell's equations for the electric and magnetic fields, $E_{y}, H_{x}$, and $H_{z}$ reduce to

$$
\begin{aligned}
\frac{\partial H_{x}}{\partial t} & =-\frac{1}{\mu_{0}} \frac{\partial E_{y}}{\partial z}, \\
\frac{\partial H_{z}}{\partial t} & =-\frac{1}{\mu_{0}} \frac{\partial E_{y}}{\partial x}, \\
\frac{\partial E_{y}}{\partial t} & =-\frac{1}{\varepsilon}\left(\frac{\partial H_{z}}{\partial x}-\frac{\partial H_{x}}{\partial z}\right),
\end{aligned}
$$

where $\mu_{0}$ and $\varepsilon$ are the magnetic and electric permeability, respectively. The FDTD simulations solve Eqs. (1)-(3) on a spatial grid for a given initial field distribution. We used the so-called unsplit perfectly matched layer (UPLM) boundary conditions [13] and a uniform discretization with a step size range of $0.02 \mu \mathrm{m}$ to $0.08 \mu \mathrm{m}$ in the $x$ and $z$ directions and with a time step range of $4.45 \times 10^{-17} \mathrm{~s}$ to $1.78 \times 10^{-16} \mathrm{~s}$.

For our calculations the initial condition was such that the disk was empty and that light was pumped into the lowest transverse mode of the linear waveguide from one end. The incoming light was either a continuous wave or a 30fs Gaussian pulse. In the former case, we are interested in the steady state field distribution which, for example, allows us to observe the resonant disk mode and the evanescent field. Pulsed input allows to calculate the output power at the other end of the linear waveguide as a function of frequency, i.e., the transmission spectrum, by applying a discrete Fourier transform on the output field, calculating the Poynting power density and integrating over the waveguide cross-section.

As an example of our FDTD simulations, snapshots in time of the propagation of a 30fs pulse are shown in Fig. 2. The pulse enters through the linear waveguide, is coupled into the microdisk cavity and completes one round trip in approximately $230 \mathrm{fs}$.

\section{B. Coupled mode theory}

We complement the numerical FDTD results with a semi-analytical CMT. For this, it is assumed that the linear waveguide supports only a single transverse mode, while the disk supports two degenerate, counterpropagating WGM. The pumped waveguide mode only couples to the forward propagating disk mode, but scattering due to side-wall roughness may couple light into the second mode. Both WGM are coupled to the waveguide mode via their evanescent fields at point 1 in Fig. 1. For the calculations we closely follow the work by Rowland and Love [9].

First, the WGM are obtained as solutions of Eqs. (1)-(3) in cylindrical coordinates. This gives mode functions 

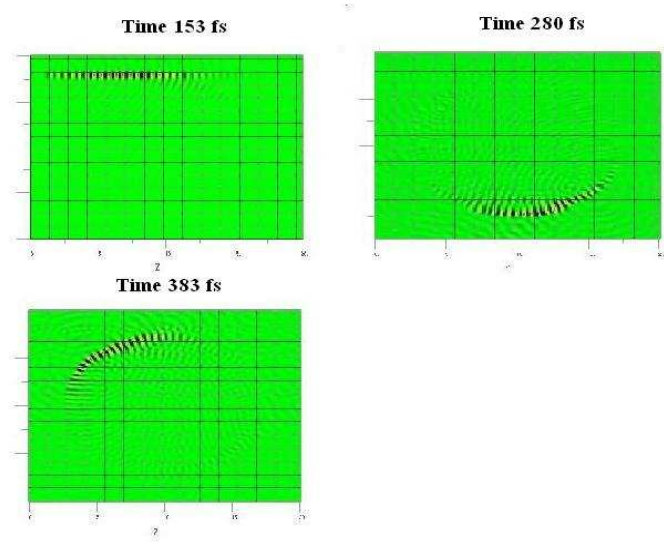

FIG. 2: FDTD computed initial coupling of $30 \mathrm{fs}$ pulse into a microdisk $(D=15 \mu \mathrm{m}, n=1.454, w=0.6 \mu \mathrm{m}$, gap size $0.1 \mu \mathrm{m}$, wavelength $0.7807 \mu \mathrm{m}$.)

of the form

$$
E_{W G M}(r, \phi)= \begin{cases}\frac{J_{l}\left(k n_{c} r\right)}{J_{l}\left(k n_{c} R\right)} e^{ \pm i l \phi} & \text { for } r<R \\ \frac{H_{l}^{(1)}\left(k n_{c l} r\right)}{H_{l}^{(1)}\left(k n_{c l} R\right)} e^{ \pm i l \phi} & \text { for } r>R\end{cases}
$$

where $J_{l}$ are Bessel functions and $H_{l}^{(1)}$ are Hankel functions of the first kind, and $R$ is the disk radius. The eigenvalue equation for these modes is given by

$$
n_{c} \frac{J_{l+1}\left(k n_{c} R\right)}{J_{l}\left(k n_{c} R\right)}=n_{c l} \frac{H_{l+1}^{(1)}\left(k n_{c l} R\right)}{H_{l}^{(1)}\left(k n_{c l} R\right)} .
$$

As all WGM are lossy, the eigenvalues are complex

$$
k=k_{r}-i k_{i}
$$

and the intrinsic quality factor of the WGM is given by [9]

$$
Q_{W G M}=\frac{k_{r}}{2 k_{i}} .
$$

Similarly, the mode functions $E_{l i n}$ of the linear waveguide are calculated for the same wavenumber $k$.

The second step of the CMT is to write the total light field as a superposition of disk and waveguide mode

$$
E(x, z)=a_{1}(z) E_{l i n}(x, z)+a_{2}(z) E_{W G M}(x, z) .
$$

The coupled mode equations read

$$
\begin{aligned}
& \frac{d a_{1}}{d z}=-i \beta_{l i n} a_{1}+i C(z) a_{2}, \\
& \frac{d a_{2}}{d z}=-i \beta_{W G M}(z) a_{2}+i C(z) a_{1}
\end{aligned}
$$

where $\beta_{\text {lin }}$ and $\beta_{W G M}$ are the propagation constants of the waveguide and the WGM, respectively, and $C(z)$ is the position-dependent coupling coefficient obtained by calculating the mode overlap of waveguide and disk. For details of this calculations see Ref. [9]. 
Finally, integrating Eqs. (9) and (10) over $z$ in the region of significant coupling around point 1 in Fig. 1 yields the coupler transmission matrix $T$ which relates the waveguide and disk output fields to the inputs,

$$
\left(\begin{array}{l}
a_{1} \\
a_{2}
\end{array}\right)_{\text {out }}=T\left(\begin{array}{l}
a_{1} \\
a_{2}
\end{array}\right)_{\text {in }}
$$

where

$$
T=\left(\begin{array}{ll}
t_{11} & t_{12} \\
t_{21} & t_{22}
\end{array}\right)
$$

The cavity decay rate (half width at half maximum) $\kappa_{T}$ of the WGM due to the coupling to the waveguide is

$$
\kappa_{T}=\left|t_{12}\right|^{2} /\left(2 T_{r}\right)
$$

where $T_{r}=2 \pi l /(c k)$ is the round trip time. The corresponding quality factor is

$$
Q_{\text {coup }}=c k /\left(2 \kappa_{T}\right)=2 \pi l /\left|t_{12}\right|^{2} .
$$

\section{OPTICAL PROPERTIES OF WAVEGUIDE-COUPLED WHISPERING GALLERY MODES}

\section{A. System losses}

Apart from the intrinsic WGM losses (7) and the coupling losses into the waveguide (14) at least two other loss mechanisms have to be taken into account, intrinsic material losses and surface scattering losses.

The main material loss mechanisms are bulk Rayleigh scattering and ultraviolet and infrared absorption. The corresponding quality factor $Q_{\text {mat }}$ can be expressed in the form $[14,15]$

$$
Q_{m a t}=\frac{2 n_{c} \pi}{\alpha \lambda}
$$

where $\alpha$ is the loss coefficient and $\lambda=2 \pi / k$ is the vacuum wavelength. Material losses for fused silica in the wavelength range near $780 \mathrm{~nm}$ are of the order of $5 \mathrm{~dB} / \mathrm{km}$, which gives rise to $Q \sim 10^{10}$.

Greater uncertainty is associated with losses due to surface scattering and absorption due to surface roughness or the presence of an absorbing impurity on the surface of the disk. For a given size of surface inhomogeneities (roughness) and correlation length, the surface scattering quality factor $Q_{\text {surf }}$ has to take into account not only the direct scattering of light out of the disk ("leakage") but also scattering into other modes with high rates of leakage. Various expressions have been used to describe quality limits due to surface scattering [14-16]. In this work, we apply the expression based on the model of Rayleigh scattering by molecule-sized surface clusters [14]

$$
Q_{\text {surf }}=\frac{D \lambda^{2}}{2 L_{c} \pi^{2} \sigma^{2}},
$$

where $D$ is the disk diameter, $\sigma$ is the root-mean-square of the surface roughness and $L_{c}$ the surface correlation length. As was reported in [17], the numerical values for $\sigma$ and $L_{c}$ can be less than $1 \mathrm{~nm}$ and $5 \mathrm{~nm}$, respectively. In our calculations we used $\sigma=1 \mathrm{~nm}, L_{c}=5 \mathrm{~nm}$ and $\sigma=2 \mathrm{~nm}, L_{c}=10 \mathrm{~nm}$.

The overall cavity quality factor taking all the loss mechanisms discussed above into account is then given by

$$
Q^{-1}=Q_{\text {coup }}^{-1}+Q_{W G M}^{-1}+Q_{m a t}^{-1}+Q_{\text {surf }}^{-1} .
$$

This is related to the cavity line width (HWHM) $\kappa$ by

$$
Q=c k /(2 \kappa)
$$

and to the cavity finesse $F$ by

$$
F=Q \frac{\mathrm{FSR}}{c k} \approx Q / l
$$

where FSR $\approx c k / l$ denotes the free spectral range ( $l$ is the mode index of the WGM). For later use we also introduce $\kappa_{\text {loss }}=\kappa-\kappa_{T}$ which is the cavity loss rate due to losses into all channels apart from the linear waveguide. 


\section{B. Results}

After describing the building blocks of our calculation and loss mechanisms, let us now discuss some numerical results for the optical properties of our system. This will serve two purposes: first, to compare our different calculation methods with each other and with available experimental data in order to show the accuracy of our results; second, we need to apply our calculation to the experimental parameters that are of interest in our case, in order to establish a base for the atom-light interaction that will be discussed in the section VI.

We have compared the spatial profile of the WGM obtained from FDTD simulation with the one resulting from a CMT calculations and found good agreement,. We have also looked at the resonance spectrum of the disk for different parameters, and extracted values for the free spectral range (FSR) and the quality factor $Q$. As presented below, here too the agreement was good.

In Fig. 3, we plot the FSR for disk diameters $D$ in the range of $5-50 \mu \mathrm{m}$, refractive index values $1.454-3.2$ and wavelengths near $0.78 \mu \mathrm{m}$ and $1.55 \mu \mathrm{m}$. The latter is chosen to compare with previously published results [12]. FDTD simulations and analytically calculated WGM are in excellent agreement with each other and with the experimental data. As expected, the FSR is approximately inversely proportional to the disk diameter.

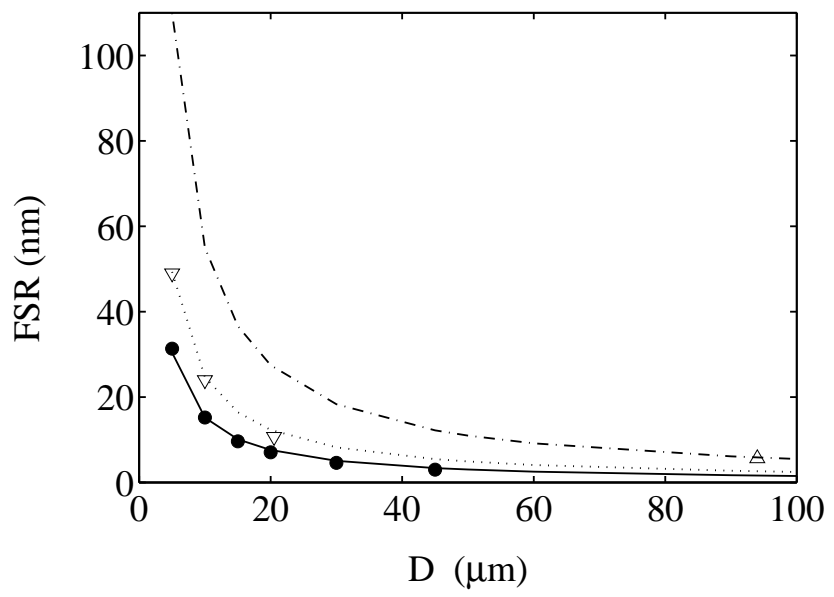

FIG. 3: Free spectral range versus disk diameter. The lines present results of analytical calculations at wavelengths $780 \mathrm{~nm}$ (solid line) and 1550nm (dashed) in fused silica and at $1550 \mathrm{~nm}$ for $n_{c}=3.2$ (dotted). FDTD results are indicated by $(\bullet)$, and experimental data $[12]$ by $(\nabla)$ and $[5]$ by $(\triangle)$.

The main result of our simulation is to show the ability to arrive at a high $\mathrm{Q}$ with relatively small disk sizes worthy of the title of micro resonators. The quality factor $Q_{\text {total }}$ was calculated by using FDTD and CMT models for different gap width depends on disk diameter. The total Q also included all loss mechanisms described in section IB. We present (Fig.3) also upper limit for quality factor in assuming ideal almost lossless coupling between straight waveguide and a disk.

Figure 4 shows the total cavity quality factor $Q$, Eq. (17), dependent on the outer diameter $D$ of the disk and the size of the air gap between disk and linear waveguide. $Q$ was calculated using FDTD and CMT models, which again show excellent agreement. We note that very high quality factors up to about $10^{8}$ can be achieved with the current system for disk diameters of several tens of microns. The upper limit for $Q$ shown in Fig. 4 is the value obtained for a very large air gap, where coupling losses $\kappa_{T}$ are negligible and the cavity quality is limited by the intrinsic and material quality factors $Q_{W G M}, Q_{m a t}$, and $Q_{s u r f}$. We have also performed calculations at wavelength $\lambda=1550 \mathrm{~nm}$ and found good agreement with recently reported $Q$ values [5].

To calculate the $Q$ factor for different disk sizes, the index $l$ of the WGM must be changed accordingly to keep the resonant wavelength near 780nm relevant for $\mathrm{Rb}$ atoms. The necessary wavelength for optimal mode resonance can be achieved by choosing a precise disk size. We will discuss tuning of the micro resonator later in Sec. V.

Let us briefly mention the issue of different refractive indices. So far,different materials have been used to 


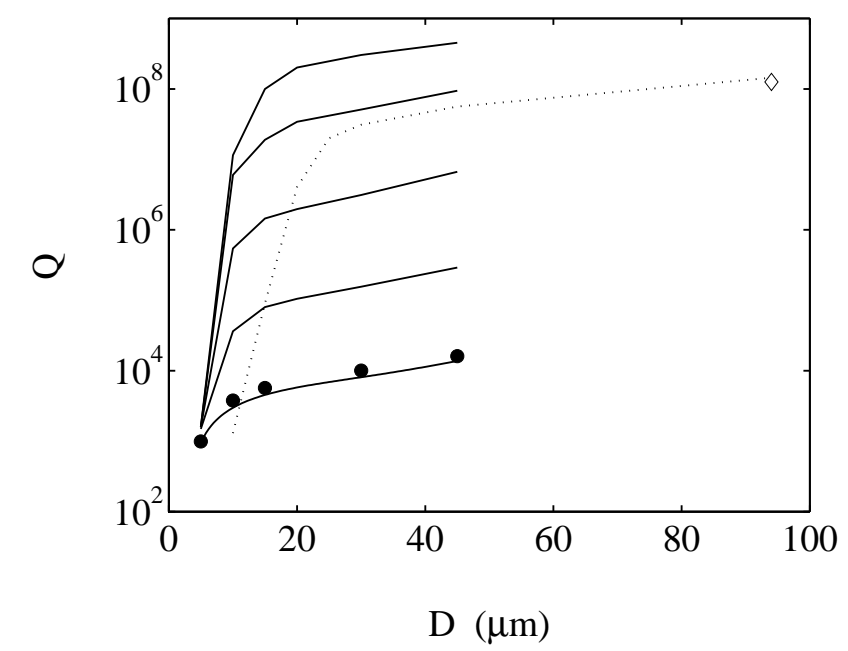

FIG. 4: Quality factor $Q$ versus disk diameter for gap sizes $0.1 \mu \mathrm{m}, 0.3 \mu \mathrm{m}, 0.5 \mu \mathrm{m}, 0.7 \mu \mathrm{m}$ (from bottom to top). The topmost curve is the quality factor $Q$ of the uncoupled disk. The solid lines present the CMT calculation and $(\bullet)$ represents the FDTD simulations. Parameters are $\lambda=780 \mathrm{~nm}, n_{c}=1.454, \sigma=2 \mathrm{~nm}, L_{c}=10 \mathrm{~nm}$. The dashed line represents the results for $\mathrm{n}=1.444$ at wavelength $1550 \mathrm{~nm}$ and $(\diamond)$ shows the measured result of [5].

fabricate microdisk resonators with refractive indices ranging from 1.444 up to $3.5[5,12,19]$. In Fig. 4 the quality factor $Q$ was presented for fused silica at different wavelengths. We checked $Q$ for materials with other refractive indices where some tuning mechanisms have been reported. Note that the $Q$ factor for higher refractive indices is even higher as shown in Fig. 5 as far as material losses not limited it.

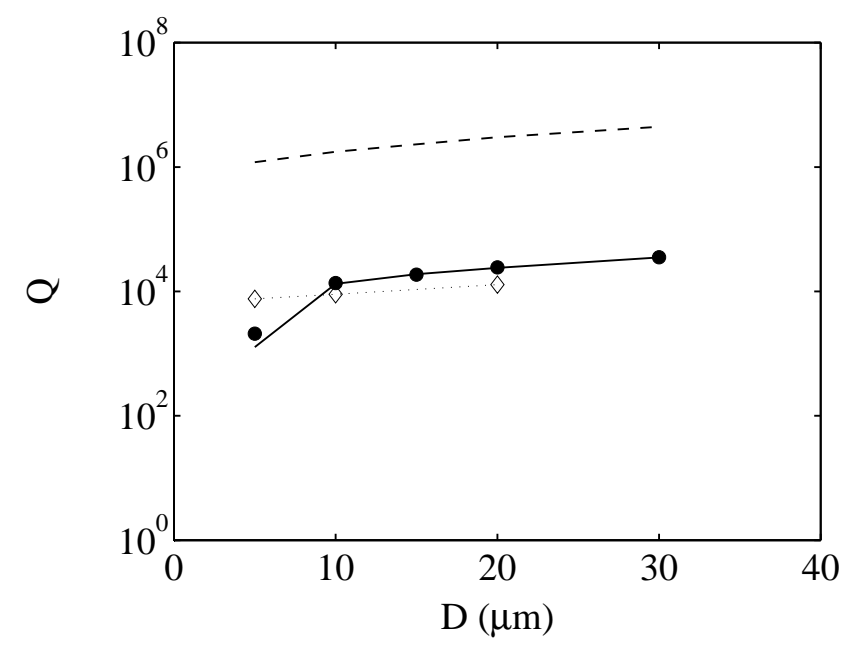

FIG. 5: Quality factor $Q_{\text {total }}$ vs. disk diameter for different refractive indexes (gap size $0.2 \mu \mathrm{m}$ ). At $780 \mathrm{~nm}$, the lines present the CMT calculation for $n=2.17$ (dashed) and $n=1.454$ (solid) and $(\bullet$ ) represents the FDTD simulation. At $1550 \mathrm{~nm}$ and $\mathrm{n}=3.2$, the dotted line shows the simulation results and $(\diamond)$ represents the experimental data of Ref.[12].

Note also that, while the intrinsic quality factor $Q_{W G M}$ is always lower for higher radial modes, the overall $Q$ can in fact be higher due to weaker coupling (smaller losses) to the linear waveguide, which is the dominating loss mechanism for most parameter regimes we are interested in. Table I shows the optical properties of a few selected disk-waveguide geometries. 


\begin{tabular}{|c|c|c|c|c|c|}
\hline$D(\mu \mathrm{m})$ & $l$ & $q$ & $\lambda(\mathrm{nm})$ & $Q_{1}$ & $Q_{2}$ \\
\hline 30 & 167 & 1 & 778.73 & $1.55 \times 10^{5}$ & $8.44 \times 10^{6}$ \\
\hline 30 & 166 & 1 & 783.27 & $1.47 \times 10^{5}$ & $8.05 \times 10^{6}$ \\
\hline 30 & 159 & 2 & 780.04 & $1.83 \times 10^{5}$ & $8.85 \times 10^{6}$ \\
\hline 15 & 81 & 1 & 780.41 & $7.66 \times 10^{4}$ & $3.82 \times 10^{6}$ \\
\hline 45 & 253 & 1 & 780.15 & $2.66 \times 10^{5}$ & $1.40 \times 10^{7}$ \\
\hline
\end{tabular}

TABLE I: Optical properties of selected WGM. $Q_{1}\left(Q_{2}\right)$ is the quality factor $Q$ for a gap size of $0.3 \mu \mathrm{m}(0.6 \mu \mathrm{m}), l$ and $q$ are the longitudinal and radial mode index, respectively. Results are obtained using CMT.

\section{EXPERIMENTAL CONSIDERATIONS AND TUNABILITY}

In this section we consider applicability of a micro disk resonator in a real experiment. This includes a tolerance of the micro disk fabrication and that the frequencies of the resonances has to be tunable. The tunable devices are essential in the different applications,for example, as tunable optical filters which are important features for dense wavelength division multiplexing (WDM) networks ([2]) or as a physical element in the optical biosensors.

As already noted, high $Q$ devices such as micro spheres, Fabry-Perot cavities, or micro disks are an extremely effective tool for the delicate manipulation and measurement of subtle quantum states, where a long lived photon interacts many times with the same atom so that a significant interaction can be achieved. However, such strong coupling requires that the device is kept on resonance with the exact frequency close to the chosen atom transition $[6,18]$.

In general, the FWHM and the FSR of microdisks differ by orders of magnitude and therefore coincidences between the transverse fundamental WGM and the atomic transition frequency are extremely unlikely. To keep a WGM resonance near the wavelength of interest we need to change the diameter or the refractive index of the disk. The tuning procedure should also be stable and reversible and the tuning range has to be of the same order of magnitude as the FSR. Under these conditions a resonant mode close to the required atomic frequency can always be found, even if the disk was initially fabricated with some mismatch in diameter or in refractive index. Finally, the tuning procedure also has to be fast enough to compensate for temporal instabilities such as those arising from temperature fluctuations of the chip.

To realize tunability for microdisk resonators one may consider at least three possibilities: UV-, optoelectricaland piezo- effects.

All of them affect, at first order, the mode resonance through simple relation [18]: $\Delta \nu / \nu=-\Delta n / n-\Delta D / D$, where $\nu$ is the resonance mode frequency, $\mathrm{n}$ is the refractive index and $\mathrm{D}$ is the disk diameter. To scan full FSR tuning we need that one FSR tuning will be equivalent to $\Delta \nu / \nu \approx 1 / l$, where $l$ is the longitudinal mode index. Actually, since higher order radial modes could also be used, there are in fact several usable resonances within each FSR.

In Fig. 6 we show the results of our calculation of relative change of the diameter and refractive index vs. disk diameter for fused silica $(\mathrm{n}=1.454$ at $\lambda=780 \mathrm{~nm})$ and for $\mathrm{n}=2.17$ at $\lambda=780 \mathrm{~nm}$, in order to scan one full FSR.

UV tuning was reported in [20]. There, it has been shown that it is possible to change the refractive index of Ge doped silica by up to $\Delta n=0.006$ using UV radiation. The drawback is that this procedure is not reversible.

In the case of electro - optical tuning, the effect is achieved by a uniform electric field that tunes the optical refractive index and allows to change the wavelength. The idea is to cover both the bottom and top of the disk with a metal layer, and apply a voltage to create the necessary electric field. For example, crystaline materials usually have a relative index of refraction change of $0.01 \%-1 \%$ for a field of $10^{6} \mathrm{~V} / \mathrm{m}$ ( $5 \mathrm{~V}$ for our $5 \mu m$ disk thickness). Though demanding a more complex fabrication process, such crystals may indeed be used. Figure 6 compares the needed values to the former 1\%, and shows that disk diameters as small as $15 \mu m$ will enable a full FSR scan. Other methods for such index of refraction changes also exist. For example, it was reported in [19] that tuning an InP micro disk was done by utilizing free carrier injection to change the refractive index from $\Delta n \sim 0.002$ up to $\Delta n \sim 0.01$. 


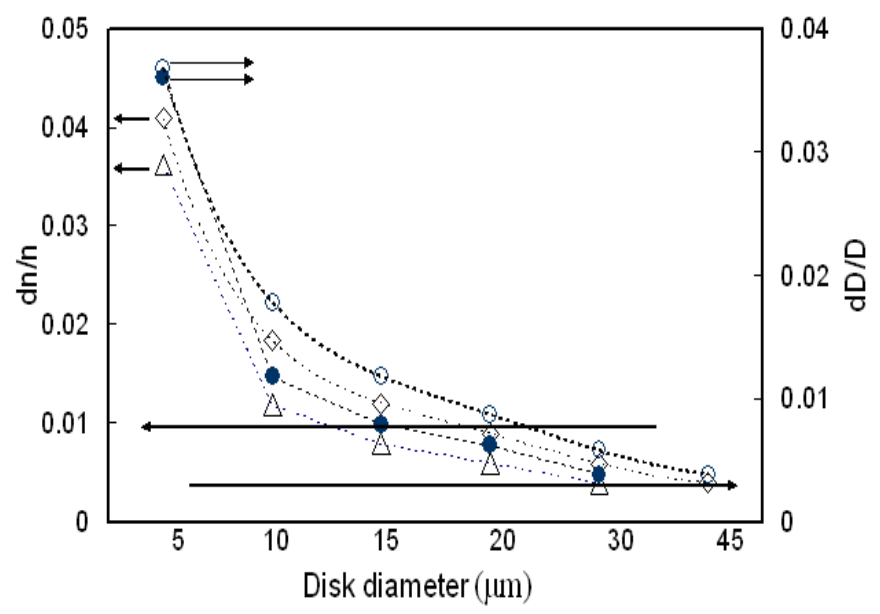

FIG. 6: Requirement for full FSR scan capability. The required relative change for fused silica of the diameter and refractive index vs. disk diameter $(\lambda=780 \mathrm{~nm}) . \Delta D / D$ and $\Delta n / n$ are presented by $\circ$ and $\diamond$ for $\mathrm{n}=1.454$, and for $\mathrm{n}=2.17$ by $\bullet$ and $\triangle$, respectively. Typical values for actual materials are also presented by the long base arrows.

By using a piezo effect one can change the diameter of the disk. Namely, one fabricates the disk from a transparent piezo material. The voltage necessary will be obtained as above by two electrodes evaporated below and above the device. Transparent piezo materials (such as $\mathrm{BaTiO}_{3}$ ) have a piezo electric coefficient of order $10^{-10} \mathrm{~m} / \mathrm{V}$ which leads to $\Delta D / D=0.002$ for $2010^{6} \mathrm{~V} / \mathrm{m}$ (100V for our $5 \mu \mathrm{m}$ disk thickness). Figure 6 again compares the needed values to the former $\Delta D / D=0.002$, and shows that disk diameters as small as $30 \mu m$ will enable a full FSR scan. For both $\Delta D / D$ and $\Delta n / n$, higher voltages and more exotic materials should allow for even smaller disk sizes.

\section{SINGLE ATOM DETECTION}

Recently it was proposed to use for the purpose of atom-light interaction very small optical cavities microfabricated on the atomchip based on Fabry-Perout [6] or photonic bandgap [7] cavities. Here, we will investigate the properties of a microdisk, modeled as a high finesse ring resonator as described in Ref. [9], as a single atom detector for quantum information processing on an atom chip. The scheme we discuss here is developed in [22] and based on Jaynes-Cummings model for the coupling of a two level atom to the light field outside the disk. Homodyne detection of the phase change of the light at the output of the linear waveguide in the forward direction is used to detect. There are several advantages of this scheme over a corresponding absorption detection. (i) It allows to drive the atom far off resonance, in which case the precise tuning of the disk resonator with respect to the atomic transition frequency is of minor importance. Cavity tuning will be discussed in more detail in Sec. V. (ii) If the additional loss mechanisms discussed in Sec. IV A are small compared to the disk-waveguide coupling strength, all of the pump light will leave the system through the forward waveguide output. Therefore for most parameter regimes a strong signal can be expected, which allows the use of standard photodetectors rather than sophisticated single-photon counters. (iii) The strong output signal also provides stability of the detection scheme against weak background scattering processes.

Our detection scheme works as follows. The output field of the straight waveguide is mixed with a strong local oscillator field at a 50-50 beamsplitter and the light intensities in the two beamsplitter outputs are measured and integrated over the observation time $\tau$ to give the total number of detected photons $N_{1}$ and $N_{2}$. The signal we are interested in is given by the difference $\left|N_{1}-N_{2}\right|$. The phase of the local oscillator is adjusted such that this difference is zero when no atom is interacting with the disk field. The presence of an atom is then inferred 
from a change in this intensity difference. Assuming that the detection is shot-noise limited, the signal-to-noise ratio $S$ of the atom detection is given by

$$
S=\frac{\left|N_{1}-N_{2}\right|}{\sqrt{N_{1}+N_{2}}} \approx 2 \sqrt{\tau}\left|A_{\text {out }, 0}\right|\left|\sin \left(\phi-\phi_{0}\right)\right|
$$

where $\phi\left(\phi_{0}\right)$ is the phase of the waveguide output field $A_{\text {out, }+}$ with (without) an atom, $A_{\text {out }, 0}$ is the field amplitude without an atom, and we have assumed that only the phase and not the amplitude of the output is changed due to the atom.

The second quantity of interest is the number of photons $M$ spontaneously scattered by the atom during the interaction time, given by

$$
M=2 \Gamma \tau \rho_{11} .
$$

where $\Gamma$ is atomic decay rate and $\rho_{11}$ is population of atomic excited state. This should be as small as possible in order to minimize the backaction of the detection process onto the atom. Ideally, $M \ll 1$ corresponds to the limit of non-destructive measurements. Finally, we note that $S$ and $M$ scale differently with interaction time $\tau$. For any given parameters, we can thus choose $\tau$ in such a way to yield a certain, fixed signal-to-noise ratio. As an example, we will in the following consider the number $M_{10}$ of spontaneously scattered photons when $\tau$ is rescaled to give $S=10$,

$$
M_{10}=100 M / S^{2}
$$

For simplicity we will assume that the cavity is driven on resonance with the disk modes, $\Delta_{c}=0$, thereby minimizing the effect of other, off-resonant modes. The atom is assumed to be far off resonance with respect to the cavity mode, $\Delta_{a} \gg \Gamma$, such that the effect of the atom is mainly to provide a phase shift of the cavity mode. Under these conditions and in the limit of small atomic saturation we can derive analytical approximations for $S, M$ and $M_{10}[6]$

$$
\begin{aligned}
S & =4 \sqrt{\tau}\left|A_{i n}\right| \frac{\kappa_{T} g^{2}}{\Delta_{a} \kappa^{2}}, \\
M & =4 \tau\left|A_{i n}\right|^{2} \frac{\kappa_{T} g^{2} \Gamma}{\Delta_{a}^{2} \kappa^{2}}, \\
M_{10} & =25 \frac{\kappa^{2} \Gamma}{\kappa_{T} g^{2}} .
\end{aligned}
$$

Note that $M_{10}$ is independent of the pump power and of the atomic detuning.

In Fig. 7 we show $S, M$ and $M_{10}$ as a function of the input power to the linear waveguide. For a weak pump the signal-to-noise ratio increases with power since more photons are coupled into the cavity and interact with the atom. However, because of saturation the atom can only interact with a maximum number of photons in a given interaction time. Hence, $S$ reaches a maximum value, and for stronger pump powers $S$ is decreasing again. The number of spontaneously scattered photons $M$ increases with pump power and finally saturates at the value $\Gamma \tau . M_{10}$ shows an approximately linear increase with pump power, indicating that the least perturbing atom detection for a given value of $S$ is achieved for low atomic saturation. There is, however, a trade-off as the required interaction time increases in this limit. For low saturation the numerical results are accurately described by the approximations (23)-(25). (For details see $[\mathrm{xx}]$.)

Figure 8 shows $S$ and $M_{10}$ as a function of the gap between the microdisk and the waveguide in the limit of weak atomic saturation $\left(\rho_{11}<0.03\right.$ for the shown parameter range). For small enough gap sizes, increasing the gap reduces the coupling between disk and waveguide modes and therefore the cavity finesse increases. This leads to improved signal-to-noise ratios and less spontaneous photon scattering by the atom. For very large gaps, on the other hand, the cavity finesse is limited by the additional losses, see Sec. IV A. In this case, increasing the gap even further reduces the number of photons coupled back from the cavity into the waveguide and thus reduces the detected signal. If the cavity-waveguide coupling exactly equals the additional losses, no light is transmitted through the waveguide at all, which leads to the points of $S=0$ and the corresponding divergence 

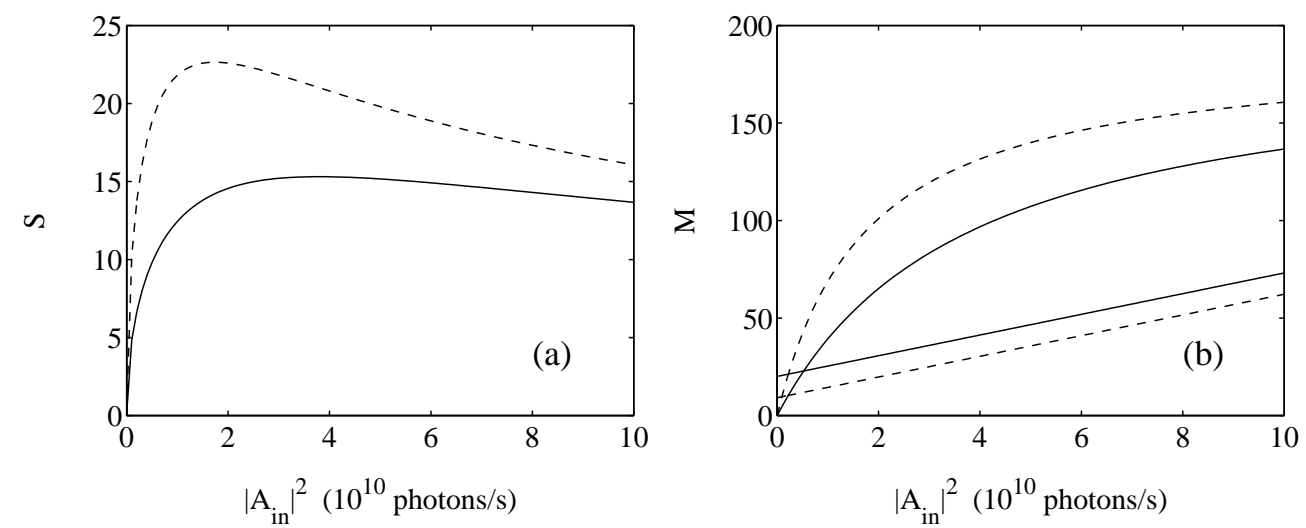

FIG. 7: (a) Signal-to-noise ratio vs pump intensity for disk diameter $30 \mu \mathrm{m}$ (solid line) and $15 \mu \mathrm{m}$ (dashed). (b) Corresponding photon scattering $M$ (top curves) and $M_{10}$ (bottom). Gap size is $0.3 \mu \mathrm{m}$, waveguide width is $0.6 \mu \mathrm{m}, \sigma=2 \mathrm{~nm}$, $L_{c}=10 \mathrm{~nm}, \Delta_{a}=100 \Gamma$, and the atom is assumed to be positioned $50 \mathrm{~nm}$ away from the disk surface.
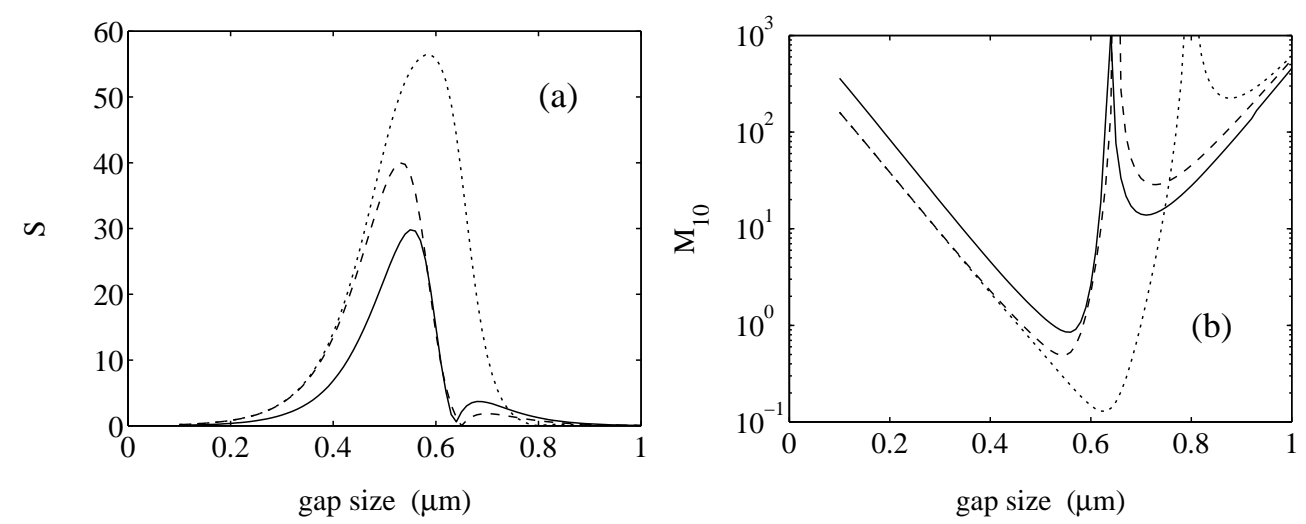

FIG. 8: (a) Signal-to-noise ratio $S$ and (b) scattered photons $M_{10}$ vs. gap size for weak pumping $\left(\left|A_{\text {in }}\right|^{2}=10^{8}\right.$ photons $\left./ \mathrm{s}\right)$. Solid curve: disk diameter $30 \mu \mathrm{m}$, dashed: disk diameter $15 \mu \mathrm{m}$ for $\sigma=2 \mathrm{~nm}, L_{c}=10 \mathrm{~nm}$. Dotted curve: diameter $15 \mu \mathrm{m}$, $\sigma=1 \mathrm{~nm}, L_{c}=5 \mathrm{~nm}$. Waveguide width is $0.6 \mu \mathrm{m}$, distance atom-disk is $50 \mathrm{~nm}$, and the interaction time is $10 \mu \mathrm{s}$.

of $M_{10}$ observed in the figure. We find numerically that $S$ is maximum and $M_{10}$ is minimum if losses from the disk into the waveguide are about 4-5 times higher than the additional losses.

For the parameters of Fig. 8 we find that the minimum value of $M_{10}$ is 0.85 for a disk diameter of $D=30 \mu \mathrm{m}$ (solid line) and 0.49 for $D=15 \mu \mathrm{m}$ (dashed line). The reason for this difference is mainly that for the smaller disk more energy of the resonant mode is in the evanescent field. This leads to improved coupling of the atom to the mode. For both disk diameters, however, the minimum value of $M_{10}$ is below unity, which indicates that single atoms can be detected while on average scattering less than one photon spontaneously. Moreover, we observe that this minimum value of $M_{10}$ is limited by the additional losses due to surface roughness. If the surface parameters are decreased by a factor of two to $\sigma=1 \mathrm{~nm}$ and $L_{c}=5 \mathrm{~nm}, M_{10}$ can be as small as 0.13, shown by the dotted curve in Fig. 8(b). In this case, our atom detection scheme approaches the limit of a non-destructive measurement. 


\section{SUMMARY AND CONCLUSION}

We have calculated the quality factor $\mathrm{Q}$ as a function of micro disk size and quality of fabrication. We have shown that it is possible to achieve a high $\mathrm{Q}$ for a micro disk diameter of several dozens of micron.

We have estimated the feasibility of initiating photon-atom interaction on an atom chip by utilizing a micro disk as a high $\mathrm{Q}$ device. We found that it is possible to achieve high signal to noise ratios with practically no photon scattering, and hence non destructive measurements may be possible.

\section{Acknowledgments}

This work was supported by the UK Engineering and Physical Sciences Research Council and the European Union, Contract No. IST-2001-38863 (ACQP). R.F. gratefully acknowledges the support of European Union FP6 'atomchip' collaboration, The German Federal Ministry of Education and Research (BMBF) through the DIP project, and the Israeli Science Foundation.

[1] R. E. Slusher, A. F. J. Levi, U. Mohideen, S. L. McCall, S. J. Paerton, and R. A. Logan, Appl. Phys. Lett. 63, 1310 (1993).

[2] D. Sadot and Efraim Boimovich, IEEE Comm. Magazine 50 (1998).

[3] F. Vollmer, D. Braun, A. Libchaber, M. Khoshsima, I. Teraoka, and S. Arnold, Appl. Phys. Lett. 80, 4057 (2002).

[4] T. Pellizzari, S. A. Gardiner, P. Zoller, Phys. Rev. Lett. 75, 3788 (1995); S. J. Van Enk, J. I. Cirac, P. Zoller, Science 279, 205 (1998); C. J. Hood, M. S. Chapman, T. W. Lynn, H. J. Kimble, Phys. Rev. Lett. 80, 4157 (1998), P.W. Pinkse, T Fisher, P Maunz and G. Rempe, Nature 404, 365 (2000).

[5] T. J. Kippenberg, S. M. Spillane, D. K. Armani, and K. J. Vahala, Appl. Phys. Lett. 83, 797 (2003); D.K. Armani, T.J. Kippenberg, S.M. Spillane, and K.J. Vahala, Nature 421, 925 (2003); V. Zwiller, S. Fälth, J. Persson, W. Seifert, L. Samuelson, and G. Björk, J. Appl. Phys. 93, 2307 (2003).

[6] P. Horak, B. G. Klappauf, A. Haase, R. Folman, J. Schmiedmayer, P. Domokos, and E. A. Hinds, Phys. Rev. A 67, 043806 (2003).

[7] B. Lev, K. Srinivasan, P. Barclay, O. Painter, and H. Mabuchi, arXiv:quant-ph/0402093 (2004).

[8] R. Stoffer, K. R. Hiremath, and M. Hammer, Proceedings of the International School of Quantum Electronics, 39th course, Erice, Sicily (October 2003); M. Hammer, K. R. Hiremath, and R. Stoffer, AIP Conference Proceedings, Melville, New York (to appear in 2004).

[9] D. R. Rowland and J. D. Love, IEE Proceedings-J 140, 177 (1993).

[10] A. I. Rahachou and I. V. Zozoulenko, arXiv:physics/0307024 (2003).

[11] T. Yamamoto and M. Koshiba, J. Lightwave Technol. 11, 400 (1993).

[12] S. C. Hagness, S. T. Ho, and A. Taflove, J. Lightwave Technol. 15, 2154 (1997); D. Rafizadeh, J.P. Zhang, S. C. Hagness, A. Taflove, K.A.Stair, S.T. Ho, and R.C. Tiberio, Optics Lett., 22, 1244 (1997).

[13] J. P. Berenger, J. Comput. Phys. 114, 185 (1994); S. D. Gedney, IEEE Trans. Antennas Propagation, 1630 (1996); C. E. Reuter, R. M. Joseph, E. T. Thiele, D. S. Katz, and A. Taflove, IEEE Microwave Guided Wave Lett. 4, 344 (1994).

[14] M. L. Gorodetsky, A. A. Savchenkov, and V. S. Ilchenko, Opt. Lett. 21, 453 (1996).

[15] D. W. Vernooy, V. S. Ilchenko, H. Mabuchi, E. W. Streed, and H. J. Kimble, Opt. Lett. 23, 247 (1998).

[16] S. Banna, D. Schieber, and L. Schächter, J. Appl. Phys. 95, 4415 (2004); B. E. Little and S. T. Chu, 21, 1390 (1996); A. I. Rahachou and I. V. Zozoulenko, arXiv:physics/0305111 (2003); D. G. Hall, Opt. Lett. 6, 601 (1981); J. J. Barroso, J. P. L. Neto, and K. G. Kostov, IEEE Trans. Plasma Sci. 31, 752 (2003).

[17] K. K. Lee, D. R. Lim, and L. C. Kimerling, Opt. Lett. 26, 1888 (2001); K. H. Guenter and P. G. Wierer, Proc. SPIE 401, 266 (1983).

[18] W. von Klitzing, R. Long, V. S. Ilchenko, J. Hare, and V. Lefevre-Seguin, New J. Phys. 3, 14 (2001).

[19] K. Djordjev, S.-J. Choi, S.-J. Choi, and P. D. Dapkus, IEEE Photon. Technol. Lett. textbf14, 828 (2002).

[20] R. A. Javris, J. D. Love, A. Durandet, G. D. Conway, and R. W. Boswell, Electron. Lett. 32, 550 (1996).

[21] A.L. Huston and J.D. Eversole, Optics Lett.14, 1104 (1993).

[22] M. Rosenblit, P. Horak, S. Helsby, and R. Folman, unpublished. 